\begin{tabular}{|c|c|}
\hline Title & Evolution of pyramid morphology during InA s(001) homoepitaxy \\
\hline Author(s) & Babu, J. Bubesh; Y oh, Kanji \\
\hline Citation & $\begin{array}{l}\text { A pplied Physics Letters, 97(7), } 072102 \\
\text { https://doi.org/10.1063/1.3481077 }\end{array}$ \\
\hline Issue Date & 2010-08-16 \\
\hline Doc URL & http:/hdl.handle.net/2115/43844 \\
\hline Rights & $\begin{array}{l}\text { Copyright } 2010 \text { A merican Institute of Physics. This article may be downloaded for personal use only. A ny other use } \\
\text { requires prior permission of the author and the A merican Institute of Physics. The following article appeared in A ppl. } \\
\text { Phys. Lett. } 97,072102 \text { (2010) and may be found at https://dx.doi.org } / 10.1063 / 1.3481077\end{array}$ \\
\hline Type & article \\
\hline File Information & A PL97-7_072102.pdf \\
\hline
\end{tabular}

Instructions for use 


\title{
Evolution of pyramid morphology during InAs(001) homoepitaxy
}

\author{
J. Bubesh Babu ${ }^{\text {a) }}$ and Kanji Yoh \\ Research Centre for Integrated Quantum Electronics, Hokkaido University, Sapporo 0608628, Japan
}

(Received 9 June 2010; accepted 29 July 2010; published online 16 August 2010)

Growth of InAs(001) homoepitaxial layer has been carried out especially at the bistable region, where the coexistence of both In-stabilized $(4 \times 2)$ and As-stabilized $(2 \times 4)$ surface reconstruction are found to be predominant. The observation of pyramid morphology in this bistable region is reported here. Atomic force microscopy studies have been performed on such pyramids. The heights of the observed pyramids vary from 12 to $26 \mathrm{~nm}$ with their bases from $3.6 \times 1.2$ to 18 $\times 6.3 \mu \mathrm{m}^{2}$. Formation of such pyramids in the bistable region is attributed to the unique anomalous As-desorption observed during the surface reconstruction. (C) 2010 American Institute of Physics. [doi:10.1063/1.3481077]

Atomic scale changes happening during the growth of III-V epitaxial layers by molecular beam epitaxy had played an important role in understanding the growth mechanism providing various information on nucleation, evolution of growth modes, defect formation, island formation, and surface reconstruction. There have been many reports explaining various surface informations during the homoepitaxial growth of III-V materials. Anisotropic mounds and island formation during the homoepitaxial growth of $\mathrm{GaAs}(001),{ }^{1-4}$ periodic elongation of terraces in InP homoepitaxy, ${ }^{5}$ straight edge formation on $\operatorname{InAs}(001)$ vicinal surface ${ }^{6}$ are few reports especially in homoepitaxy. The formation of such mounds, terrace, and islands, in general, the morphology of epitaxial films as the result of three-dimensional (3D) nucleation are mainly triggered by many parameters like V/III flux, substrate temperature, deposition rate, surface reconstruction, and the thickness of the growing epilayer.

Among the various III-V compound semiconductors, InAs is attracted for long-wavelength, high carrier mobility, ${ }^{7}$ high speed, and high frequency applications. ${ }^{8}$ Epitaxial thin films for post complementary metal-oxide-semiconductor digital integrated circuits ${ }^{9}$ and high performance spin devices ${ }^{10-12}$ require the surfaces to be atomically smooth with low defect densities in critical conditions. However the formation of 3D structures or islands on the III-V surfaces is found to be an important study to explore the growth mechanism. The effects of such surface modifications have shown some confinement on characterizing such surfaces. ${ }^{13}$ On the other hand, investigation on the surface morphology remains important on realization of high quality heterostructures. There have been few theoretical investigations on InAs(001) surface to elucidate the growth mechanism. ${ }^{14-18}$ Relatively few experimental work on the InAs(001) surface were done which mainly concentrate on the surface reconstruction. ${ }^{19-23}$ Bell et al. ${ }^{24}$ have investigated the islands and defects formation on $\operatorname{InAs}(001)$ surfaces, whereas the growth was carried out at low temperatures. In this letter, we report mainly the analysis of InAs surfaces as a function of substrate temperature especially at high temperatures around $(2 \times 4)$ to $(4$ $\times 2$ ) surface reconstruction. Previous reports on the growth of InAs layer at higher temperatures $465-510{ }^{\circ} \mathrm{C}$ close to $(2 \times 4)$ to $(4 \times 2)$ transition is known for its higher mobility and have shown promising device performances. ${ }^{7,25}$ How-

${ }^{a)}$ Electronic mail: bubeshbabu@yahoo.com. ever the growths beyond $510{ }^{\circ} \mathrm{C}$ for particular As-pressure taken up in this study, falls in the bistable region displays pyramid morphology. Meanwhile retaining a smallest interface roughness during the growth of InAs channel at high temperatures, by adjusting the V/III ratio have demonstrated some highest mobility upto $32000 \mathrm{~cm}^{2} / \mathrm{V} \mathrm{s}$, which is significant for device applications. ${ }^{26}$

A typical first order phase transition between Instabilized $(4 \times 2)$ region and As-stabilized $(2 \times 4)$ region was observed for InAs(001) surface. It experiences a hysteresis on increasing and decreasing the temperature of the substrate between $(2 \times 4)$ and $(4 \times 2)$ regions. ${ }^{21,22}$ Moreover the boundary between the two major regions is not sharp but separated with a band of transition temperatures, where the transition band holds a bistable phase with both $(2 \times 4)$ and $(4 \times 2)$ phases. ${ }^{6,22}$ Oxide removal process of InAs substrate and the growth chamber are exactly same as reported in our previous publication. ${ }^{12}$ With reference to the surface reconstruction diagram, 300-nm-thick InAs layer was grown under As-pressure with a beam-equivalent-pressure ratio of As to In of about 12. The As-pressure is almost between 5.8 $\times 10^{-6}$ to $6.2 \times 10^{-6}$ Torr throughout all the growth experiments. Each growth was carried out with different substrate temperatures from 450 to $525{ }^{\circ} \mathrm{C}$ as shown in Fig. 1. The G1 to G6 shown in Fig. 1 represent the growth conditions employed for the growth of InAs surfaces. Among the above growth conditions, especially G1 and G2, grown at the bistable region [In-stabilized $(4 \times 2)$ and As-stabilized $(2 \times 4)]$ are taken up in this study. The evolution of pyramid

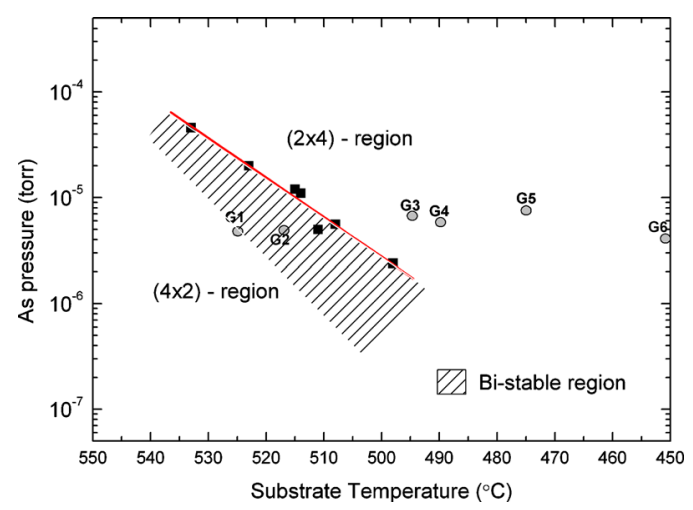

FIG. 1. (Color online) Different growth points with surface reconstruction diagram for different As-pressures. 


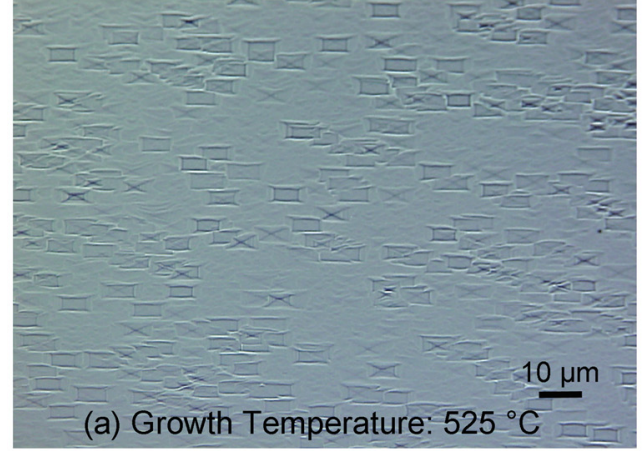

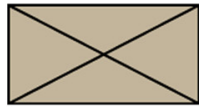

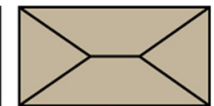

(b)
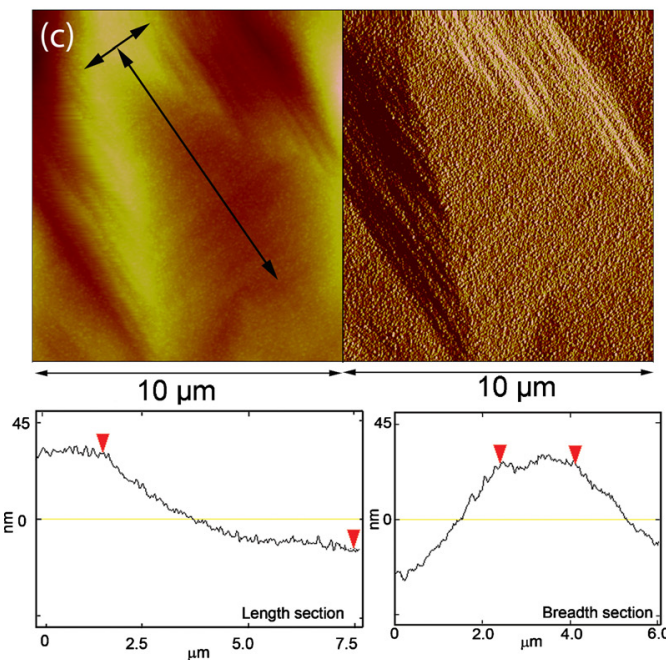

(d)

FIG. 2. (Color online) (a) Microscopic picture of InAs surface. (b) Schematic diagram. (c) AFM image. (d) AFM Section analysis of the pyramid observed for the growth conditions G1 marked in Fig. 1.

morphology observed during the growth of InAs at the bistable region has been analyzed with a LASER microscope (Keyence Voilet LASER color 3D profile microscope VK9510) and atomic force microscope (AFM) (Nanoscope IIIa) operated in contact mode. The native oxide removal temperature, growth temperature, and indium desorptiontemperature all happen to be in a narrow temperature range for InAs which make this investigation a more essential one. We conducted the surface reconstruction experiments and the InAs growth in the same growth chamber to avoid the measurement errors. Our surface reconstruction behavior satisfies well with the previous reports. 22,27

Three types of pyramid structures were observed on the surface grown at $525{ }^{\circ} \mathrm{C}$ (marked as G1) in the edge of the bistable region as shown in Figs. 2(a) and 2(b). These truncated pyramids with flat and edge terrace are observed only in this case and found to be more in number than the welldefined ones. The mechanism behind the formation of such pyramid morphology is discussed in the latter part. Figure 3(a) is the surface grown at $517{ }^{\circ} \mathrm{C}$ (marked as G2) exactly in the bistable region, where the surface has only welldefined pyramids having smaller dimensions compared to the previous case. Remaining InAs surfaces grown between 490 to $470{ }^{\circ} \mathrm{C}$ in As-stabilized $(2 \times 4)$ region (marked as $\mathrm{G} 3, \mathrm{G} 4$, and G5 in Fig. 1) do not show any pyramid morphology as
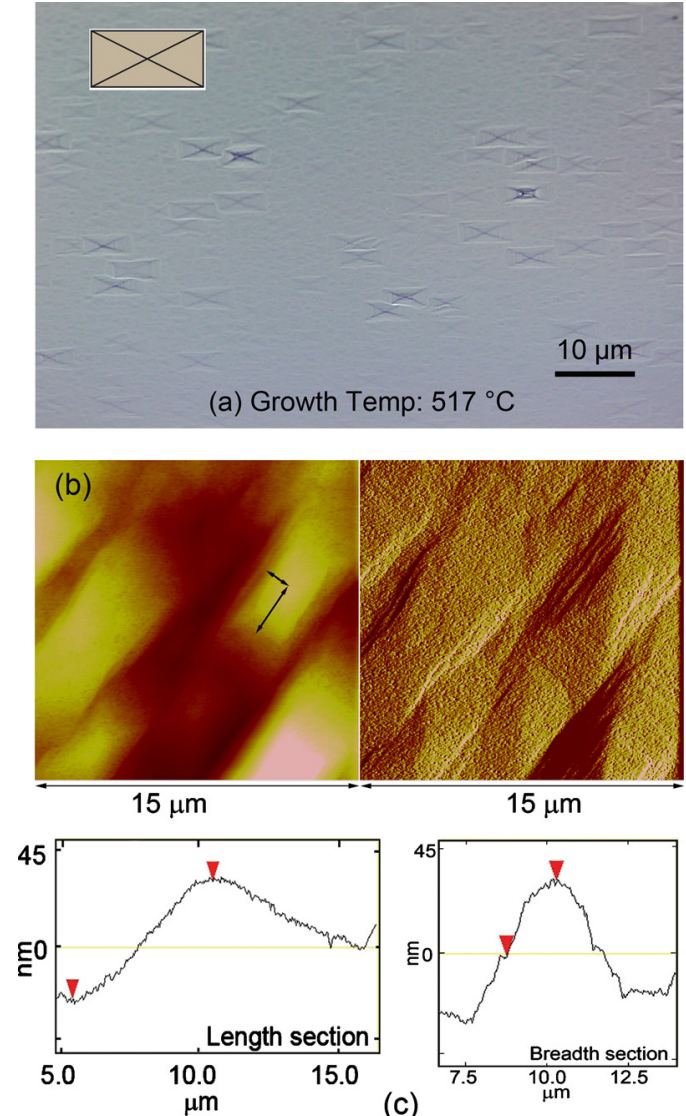

FIG. 3. (Color online) (a) Microscopic picture of InAs surface with schematic diagram of well-defined pyramid as inset. (b) AFM image. (c) AFM section analysis of the pyramid observed for the growth conditions G2 marked in Fig. 1.

observed in the pervious cases. However the surface remains rough with rms surface roughness of these samples ranging from $8.7 \mathrm{~nm}$ to $3.7 \mathrm{~nm}$, respectively. The surface grown at $450{ }^{\circ} \mathrm{C}$ in As-stabilized $(2 \times 4)$ region (marked as G6 in Fig. 1) shows a smoother and flat surface with rms surface roughness less than $1.5 \mathrm{~nm}$. To make sure that the surface defects of the substrate has no influence on the resultant pyramid morphology in G1, a separate experiment was carried out with the growth of buffer layer at $450{ }^{\circ} \mathrm{C}$ and followed by the usual growth at $525{ }^{\circ} \mathrm{C}$, which also resulted with similar pyramid morphology as observed in the samples without InAs buffer layer. For the attempts with growth temperature higher than $525{ }^{\circ} \mathrm{C}$ has resulted with indium desorption along with pyramid morphology is not presented here.

The pyramids observed at different growth temperatures in bistable region G1 and G2 [Figs. 2(a) and 3(a)] were analyzed using the Atomic force microscopy and the results were shown in Figs. 2(c) and 3(b), respectively. Figure 2(c) shows the truncated pyramids and Fig. 3(b) shows the well defined pyramids. The observed pyramids were subjected to section analysis [Figs. 2(d) and 3(c)] to measure their basic length and breadth dimensions. The length to breadth ratio of the all these pyramids observed in this study are approximately 3:1 irrespective of their types. The height of the pyramids vary from 26 to $12 \mathrm{~nm}$ with their bases are around 18 $\times 6.3$ and $3.6 \times 1.2 \mu \mathrm{m}^{2}$ for G1 and G2, respectively. Irrespective of the growth temperature, all the pyramid formations were extended along [110] direction, whereas the density of the pyramids is found to be less for $\mathrm{G} 2$ compared with 
G1. The average terrace widths of each step along the breadth and length side of the pyramids are found to be around $260 \mathrm{~nm}$ and $750 \mathrm{~nm}$, respectively, with periodic step bunching to an approximate height of $2 \mathrm{~nm}$. As the number of steps along the pyramid face are accounted approximately, these dimensions are roughly estimated to add more details on the observed pyramids.

The evolution of 3D structures (pyramid morphology) in the bistable transition region can be explained in the following manner. First of all the formation of these pyramids is observed only in the bistable region between 510 to $525{ }^{\circ} \mathrm{C}$. This can be attributed as a result of coexistence of both Instabilized $(4 \times 2)$ and As-stabilized $(2 \times 4)$ surface. $^{6}$ Ratsch et al. ${ }^{14}$ and Carter et al. $^{28}$ have done a detailed As-desorption investigations using density functional theory (DFT) calculations and kinetic Monte Carlo simulations along with their scanning tunneling microscope studies and confirmed that the surface reconstruction between $(2 \times 4)$ and $(4 \times 2)$ surfaces can further move from initial As-rich $\beta 2(2 \times 4)$ structure to In-stabilized $(4 \times 2)$ structure through an intermediate partial As-desorbed $\alpha 2(2 \times 4)$ structure. ${ }^{29}$ This $\beta 2(2 \times 4)$ and $\alpha 2(2 \times 4)$ are basically similar one with different Asdesorption rate during the surface reconstruction. Hence the $\alpha 2(2 \times 4)$ and $(4 \times 2)$ structures form a two-domain mixed surface(bistable region) with the change in surface energy which might limit the migration of In adatoms leading to formation of 3D structures. Moreover the formation of 100 $\mathrm{nm}$ step edge observed by Ohkouchi et al. as a result of $(4 \times 2)$ reconstruction may also happen partially in the present case. This may affect the migration of In atoms in the overall surface and limit the growth in the $\alpha 2(2 \times 4)$ surface. Further the elongations of the observed pyramids along [110] directions are also in a similar way as step edge directions observed by Ohkouchi et al. ${ }^{6}$

The transition between $\alpha 2(2 \times 4)$ and $(4 \times 2)$ happens with anomalous As-desorption as explained with the following desorption rate equation:

$$
\Gamma=v \exp \left(-\mathrm{E} / \mathrm{k}_{\mathrm{B}} \mathrm{T}\right)
$$

where $\Gamma$ is the desorption rate, $T$ is the surface temperature, and $\mathrm{k}_{\mathrm{B}}$ is the Boltzmann constant. Desorption prefactor, $v$, was found to be $1.5 \times 10^{19} \mathrm{~s}^{-1}$ for InAs surface and this desorption prefactor for other III-V materials are usually around $10^{13} \mathrm{~s}^{-1} \cdot{ }^{29,30}$ In the above equation desorption prefactor and $\mathrm{T}$, the substrate temperature are key parameters determining the desorption rate. The $\beta 2(2 \times 4)$ to $\alpha 2(2 \times 4)$ and $\alpha 2(2 \times 4)$ to $(4 \times 2)$ surface transitions take place with the different As-desorption rates with the desorption prefactor around $10^{17} \mathrm{~s}^{-1}$ and $10^{19} \mathrm{~s}^{-1}$, respectively. Such a unique anomalous As-desorption rate observed in InAs might be a key reason for the formation of such morphology. So the contributions of In-migration due to surface diffusionkinetics and anomalous As-desorption in the transition region have played a key role in the formation of pyramids. Surface roughening was observed in the temperature range of $470-490{ }^{\circ} \mathrm{C}$, where $\beta 2(2 \times 4)$ to $\alpha 2(2 \times 4)$ transition is predominant with least As-dimer defects and island formation. The As-dimer defects as a result of $\beta 2(2 \times 4)$ to $\alpha 2(2 \times 4)$ transition accompanied with unimolecular intermediate Asdesorption rate have been investigated in detail both experimentally and theoretically. $6,15,16,24,29$
In summary, the growth of InAs epitaxy in the bistable region of $(4 \times 2)$ and $(2 \times 4)$ surface has revealed the formation of pyramid morphology. The effect of surface roughening in $\beta 2(2 \times 4)$ to $\alpha 2(2 \times 4)$ transition region is attributed with the nominal As-desorption observed in this region. The pyramid morphology of InAs surface has been analyzed with AFM and the possible mechanism behind the formation of such pyramids were discussed. Formation of such pyramids in the bistable region during $\alpha 2(2 \times 4)$ to $(4 \times 2)$ transition might be as the result of unique anomalous As-desorption observed in the InAs. Hence such surface information on InAs in a narrow temperature would be highly useful in realization of high performance heterostructures.

The authors are grateful to Takeshi Ejiri, Takashi Matsuda, and Keita Konishi for their kind support during the experiments. This work was partly supported by a Grant-inAid for Scientific Research from the Japanese Ministry of Education, Culture, Sports, Science and Technology.

${ }^{1}$ V. R. Coluci, M. A. Cotta, C. A. C. Mendonca, K. M. I. Landers, and M. M. G. De Carvalho, Phys. Rev. B 58, 1947 (1998).

${ }^{2}$ M. D. Johnson, C. Orme, A. W. Hunt, D. Graff, J. Sudijono, L. M. Sander, and B. G. Orr, Phys. Rev. Lett. 72, 116 (1994).

${ }^{3}$ A. R. Avery, C. M. Goringe, D. M. Holmes, J. L. Sudijono, and T. S. Jones, Phys. Rev. Lett. 76, 3344 (1996).

${ }^{4}$ C. Orme, M. D. Johnson, J. L. Sudijono, K. T. Leung, and B. G. Orr, Appl. Phys. Lett. 64, 860 (1994).

${ }^{5}$ M. A. Cotta, R. A. Hamm, T. W. Staley, S. N. G. Harriott, M. B. Panish, and H. Temkin, Phys. Rev. Lett. 70, 4106 (1993).

${ }^{6}$ S. Ohkouchi and N. Ikoma, Jpn. J. Appl. Phys., Part 2 33, L1700 (1994). ${ }^{7}$ B. R. Bennett, B. P. Tinkham, J. B. Boos, M. D. Lange, and R. Tsai, J. Vac. Sci. Technol. B 22, 688 (2004).

${ }^{8}$ T. Akazaki, T. Enoki, K. Arai, and Y. Ishii, Solid-State Electron. 38, 997 (1995).

${ }^{9}$ D. H. Kim and J. A. del Alamo, Proceedings of the IEEE International Electron Devices Meeting, Washington, D.C., 10-12 December (IEEE, New York, 2007), pp. 629-632.

${ }^{10}$ Y. B. Xu, E. T. M. Kernohan, D. J. Freeland, A. Ercole, M. Tselepi, and J. A. C. Bland, Phys. Rev. B 58, 890 (1998).

${ }^{11}$ A. Filipe, A. Schuhl, and P. Galtier, Appl. Phys. Lett. 70, 129 (1997).

${ }^{12}$ M. Ferhat and K. Yoh, Appl. Phys. Lett. 90, 112501 (2007).

${ }^{13}$ M. A. Cotta, R. A. Hamm, S. N. G. Chu, L. R. Harriott, and H. Temkin, J. Appl. Phys. 75, 630 (1994).

${ }^{14}$ C. Ratsch, W. Barvosa-Carter, F. Grosse, J. H. G. Owen, and J. J. Zinck, Phys. Rev. B 62, R7719 (2000).

${ }^{15}$ F. Grosse and M. F. Gyure, Phys. Rev. B 66, 075320 (2002).

${ }^{16}$ F. Grosse, W. Barvosa-Carter, J. J. Zinck, M. Wheeler, and M. F. Gyure, Phys. Rev. Lett. 89, 116102 (2002).

${ }^{17}$ F. Grosse and M. F. Gyure, Phys. Status Solidi B 234, 338 (2002).

${ }^{18}$ M. Itoh, Prog. Surf. Sci. 66, 53 (2001).

${ }^{19}$ J. M. Moison, C. Guille, and M. Bensoussan, Phys. Rev. Lett. 58, 2555 (1987).

${ }^{20}$ H. Yamaguchi and Y. Horikoshi, Phys. Rev. B 45, 1511 (1992).

${ }^{21}$ H. Yamaguchi and Y. Horikoshi, Phys. Rev. Lett. 70, 1299 (1993).

${ }^{22} \mathrm{H}$. Yamaguchi and Y. Horikoshi, Appl. Phys. Lett. 64, 2572 (1994).

${ }^{23}$ H. Yamaguchi and Y. Horikoshi, Phys. Rev. B 51, 9836 (1995).

${ }^{24}$ G. R. Bell, M. Itoh, T. S. Jones, B. A. Joyce, and D. D. Vvedensky, Surf Sci. 433-435, 455 (1999).

${ }^{25}$ K. Yoh, T. Moriuchi, and M. Inoue, IEEE Trans. Electron Devices 11, 526 (1990).

${ }^{26}$ N. Kuze, H. Goto, S. Matsuno, M. Matsui, and I. Shibasaki, Microelectron. Eng. 43-44, 185 (1998).

${ }^{27}$ A. S. Bracker, M. Jyang, B. R. Bennett, J. C. Culbertson, and W. J. Moore, J. Cryst. Growth 220, 384 (2000).

${ }^{28}$ W. Barvosa-Carter, R. S. Ross, C. Ratsch, F. Grosse, J. H. G. Owen, and J. J. Zinck, Surf. Sci. 499, L129 (2002).

${ }^{29}$ F. Grosse, W. Barvosa-Carter, J. J. Zinck, and M. F. Gyure, Phys. Rev. B 66, 075321 (2002).

${ }^{30}$ C. Sasaoka, Y. Kato, and A. Usui, Appl. Phys. Lett. 62, 2338 (1993). 